Résumés des conférences et travaux

\title{
Histoire des universités médiévales et modernes
}

Jacques Verger

\section{OpenEdition \\ Journals}

Édition électronique

URL : https://journals.openedition.org/ashp/1002

DOI : $10.4000 /$ ashp.1002

ISSN : 1969-6310

Éditeur

Publications de l'École Pratique des Hautes Études

\section{Édition imprimée}

Date de publication : 2 février 2011

Pagination : 168-170

ISSN : 0766-0677

\section{Référence électronique}

Jacques Verger, "Histoire des universités médiévales et modernes ", Annuaire de l'École pratique des hautes études (EPHE), Section des sciences historiques et philologiques [En ligne], 141 | 2011, mis en ligne le 24 février 2011, consulté le 06 juillet 2021. URL : http://journals.openedition.org/ashp/1002 ; DOI : https://doi.org/10.4000/ashp.1002 


\title{
HISTOIRE DES UNIVERSITÉS MÉDIÉVALES ET MODERNES
}

\author{
Directeur d'études : M. Jacques Verger, \\ correspondant de l'Institut
}

Programme de l'année 2008-2009: I. Unité et diversité des universités médiévales et modernes. - II. Étude de documents.

Quoique un peu perturbée par le long mouvement de protestation qu'ont connu les universités de mars à mai 2009, l'année 2008-2009, qui a été la dernière de la conférence, le directeur d'études ayant cessé son activité à l'École à l'issue de cette année universitaire, a été consacrée à esquisser un tableau de la diversité des institutions habituellement regroupées, du XIII ${ }^{\mathrm{e}}$ au XVI ${ }^{\mathrm{e}}$ siècle, sous l'appellation d'universités ou de studia generalia. Certes, il existe une terminologie assez uniforme qui s'est fixée dès le XIII ${ }^{\mathrm{e}}$ siècle, comme l'a bien montré Olga Weijers, et cette uniformité lexicale renvoie elle-même à une certaine unité institutionnelle et juridique qui s'exprime à travers les notions d'autonomie, d'universalité et d'excellence du savoir, de collation enfin de diplômes reconnus dans l'ensemble de l'orbis christianus; ces notions permettent de distinguer clairement les « universités » de toutes les autres formes antérieures ou contemporaines d'enseignement. Elles ne doivent cependant pas cacher le fait que, dans la réalité, les institutions réunies sous cette appellation étaient extraordinairement diverses et que l'application d'un schéma unique ne peut que masquer cette diversité historique concrète. Cette diversité s'observe aussi bien au niveau de la chronologie (ce qui pose le problème de la périodisation de l'histoire universitaire) que de la géographie (y a-t-il dès le Moyen Âge des variantes nationales ou régionales?), des nombres (grandes et petites universités), du recrutement social (universités plus ou moins aristocratiques, plus ou moins cléricales), du rapport au pouvoir (les universités des monarchies nationales et celles des cités-États), de l'orientation intellectuelle (universités à dominante philosophique, théologique, juridique, médicale), institutionnelle enfin (opposition traditionnelle de l'universitas magistrorum parisienne et de l'universitas scolarium bolognaise).

Cette problématique a été abordé à travers plusieurs études de cas qui ont permis de s'interroger sur quelques facettes de cette diversité.

On s'est d'abord intéressé au problème des «petites universités », i. e. des studia generalia de fondation tardive, de rayonnement purement local, aux effectifs réduits voire squelettiques (deux ou trois maîtres, quelques dizaines d'étudiants), à l'existence parfois éphémère - mais parfois aussi durable - et cependant pourvus de tous les privilèges nécessaires, conférés par l'autorité pontificale et impériale, et généralement soutenus, au moins initialement, par un pouvoir seigneurial ou municipal local. Faut-il parler d'échec, résultant d'une mauvaise appréciation des attentes et des 
besoins sociaux ou ces fondations mineures avaient-elles malgré tout un sens et une utilité, aussi bien pour les autorités qui les fondaient que pour les professeurs et étudiants qui les fréquentaient et y prenaient des grades sur la valeur desquels il est pourtant légitime de s'interroger?

Ont été ainsi étudiés successivement le cas de l'université d'Orange, studium generale né des efforts conjoints de la Commune et du prince d'Orange, sanctionnés par un diplôme impérial (1365) et une bulle pontificale (1379), dont l'existence presque fantomatique se prolongera cependant jusqu'à la fin de l'Ancien Régime, grâce à la collation des grades plus qu'à des enseignements effectifs, puis celui du studium de Nîmes que les consuls, croyant pouvoir tirer profit des difficultés des universités voisines d'Avignon et Montpellier (en raison des débuts du Grand Schisme et des révoltes urbaines de la fin du règne de Charles $\mathrm{V}$ ), essayèrent de transformer en université en attirant maîtres et étudiants et en obtenant une bulle pontificale.

On a ensuite détaillé plusieurs exemples italiens, dont la spécificité est évidente par rapport au paradigme parisien de l'universitas magistrorum, de nature fondamentalement ecclésiastique et tournée avant tout vers les études philosophiques et théologiques.

Le cas de Sienne a été analysé à partir d'une présentation détaillée et critique du livre de Peter Denley, Commune and Studio in Late Medieval and Renaissance Siena, CLUEB, Bologne, 2006, XVII-495 p., complétée par la lecture d'un certain nombre de textes typiques de la documentation italienne (municipale et notariale) publiée dans G. Minucci et L. Košuta, Lo Studio di Siena nei secoli XIV-XVI. Documenti e notizie biografiche, Milan, 1989, et P. Nardi, L'insegnamento superiore a Siena nei secoli XI-XIV. Tentativi e realizzazioni dalle origini alla fondazione dello studio generale, Milan, 1996. Cette analyse a montré que si le recrutement social des maîtres et des étudiants, le système institutionnel et le rapport au pouvoir (essentiellement communal, incarné ici par les Savi allo studio, et fort peu ecclésiastique), le dispositif des cursus dans une université italienne «moyenne » comme celle de Sienne, étaient bien spécifiques par rapport à ce qu'on trouve au-delà des Alpes, la nature même de la documentation utilisée et la tradition historiographique locale avaient sans doute exagéré cette spécificité.

La situation italienne a été également illustrée par deux autres exemples : d'abord, celui de la «première université d'État» (selon la formule excessive d'E. Kantorowicz), celle de Naples, dont on a relu le diplôme de fondation par Frédéric II (1224) et ceux de réforme de Charles I ${ }^{\text {er }}$ d'Anjou (1266) et de Jeanne II (1428); ensuite celui de l'université de Rome (Studium Urbis) pour laquelle la publication récente des statuts du Collège Nardini, datés de 1484 (dans Anna Esposito et Carla Frova, Collegi studenteschi a Roma nel Quattrocento. Gli statuti della 'Sapienza Nardini', Rome, 2008) a permis d'évoquer cette institution universitaire typique de la Renaissance italienne que sont les grandes Case della Sapienza, à la fois collèges et centres de l'administration et de l'enseignement universitaires, souvent logées dans de somptueux bâtiments.

Au cours de cette première partie du séminaire, une séance particulière a été consacrée à une présentation critique détaillée de la thèse d'histoire du droit, tout récemment soutenue, de $\mathrm{M}^{\text {lle }}$ Marie Bassano, «Dominus domini mei dixit... Enseignement du 
droit et construction d'une identité des juristes et de la science juridique. Le studium d'Orléans ( ca 1230-ca 1320) »; une autre séance a permis à un auditeur régulier du séminaire, M. Thierry Kouamé, maître de conférences à l'université Paris-I (PanthéonSorbonne), de présenter, dans le droit fil de la problématique générale de cette année, celle d'une étude comparée des systèmes universitaires de l'Europe médiévale, un exposé sur «Le fonctionnement des congregationes à Paris et dans les universités anglaises (Oxford et Cambridge) aux XIII ${ }^{\mathrm{e}}$ et XIV ${ }^{\mathrm{e}}$ siècles ».

Enfin, les dernières séances de l'année ont été consacrées à esquisser le devenir des modèles universitaires médiévaux à l'époque moderne, au moins au XVI ${ }^{\mathrm{e}}$ siècle où aux traditions institutionnelles et intellectuelles divergentes héritées du Moyen Âge viennent s'ajouter les effets de la rupture confessionnelle et du renforcement des États.

On a ainsi d'abord étudié la création par Jean Sturm du gymnase de Strasbourg et sa transformation progressive, de 1538 à 1621, par la double action du magistrat municipal et de l'empereur, en une académie puis une véritable université (à partir des documents publiés dans M. Fournier et Ch. Engel, Les statuts et privilèges des universités françaises depuis leur fondation jusqu'en 1789, t. IV, Paris, 1894).

La dernière séance a permis d'évoquer brièvement la première esquisse de mondialisation de l'institution universitaire à travers l'analyse des textes relatifs à la création des premières universités extra-européennes, celles de Saint-Domingue (1538) et de Lima (1551, avec confirmation pontificale en 1571), par l'action conjuguée de l'ordre dominicain, de l'empereur Charles Quint et de la papauté, selon un schéma qui restait conforme au modèle médiéval de Salamanque, mais avec des perspectives coloniales et missionnaires clairement affichées (textes publiés par A. M. Rodriguez Cruz, Colección documental. Selección de algunos de los documentos más importantes de la historia en la Universidad de Salamanca y de su proyección en Hispanoamérica, Salamanque, 1977). 